Paolo Dai Pra · Sylvie Roelly · Hans Zessin

\title{
A Gibbs variational principle in space-time for infinite-dimensional diffusions
}

Received: 20 May 1999 / Revised version: 14 May 2001 /

Published online: 11 December 2001 - (C) Springer-Verlag 2001

Abstract. We show that the set of stationary weak solutions for a class of infinite dimensional stochastic differential equations coincides with the set of shift invariant, space-time Gibbs fields for a certain potential. The key step consists in proving the Gibbs variational principle for space-time Gibbs fields.

\section{Introduction}

Let $\Omega=\mathcal{C}(\mathbb{R}, \mathbb{R})^{\mathbb{Z}^{d}}$ be the configuration space, and $\mathcal{F}$ be the canonical $\sigma$-field in

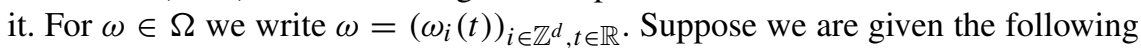
infinite-dimensional stochastic differential equation (s.d.e.)

$$
d X_{i}(t)=\left(-\frac{1}{2} \varphi^{\prime}\left(X_{i}(t)\right)+\mathbf{b}\left(\theta_{i, t} X\right)\right) d t+d B_{i}(t), \quad i \in \mathbb{Z}^{d}, t \in \mathbb{R}
$$

where

- $\varphi$ is a suitable self potential, to be chosen in a class that will be defined later (see Section 4);

- b : $\mathcal{C}((-\infty, 0], \mathbb{R})^{\mathbb{Z}^{d}} \rightarrow \mathbb{R}$ is a measurable bounded local function, say $\mathbf{b}(\omega)=$ $\mathbf{b}\left(\omega_{\Lambda_{0}}\right)$, where $\omega_{\Lambda_{0}}$ is the restriction of $\omega$ to a finite subset $\Lambda_{0} \subset \mathbb{Z}^{d}$ of spacecoordinates, containing the origin;

- $\theta_{i, t}$ is the space-time translation on $\Omega$ given by $\left(\theta_{i, t} \omega\right)_{j}(s)=\omega_{i+j}(t+s)$;

- $\left(B_{i}\right)_{i \in \mathbb{Z}^{d}}$ is a sequence of independent real valued Brownian motions.

This research was partly supported by MURST Grant "Stochastic Processes with Spatial Structure".

P. Dai Pra: Dipartimento di Matematica Pura e Applicata Università di Padova, Via Belzoni 7, 35131 Padova, Italy.

S. Roelly: Centre de Mathématiques Appliquées, École Polytechnique, 91128 Palaiseau Cedex, France.

Present address: WIAS, Mohrenstrasse 39, 10117 Berlin, Germany

e-mail: roelly@wias-berlin.de.

H. Zessin: Fakultät für Mathematik, Universität Bielefeld, 33501 Bielefeld, Germany

Mathematics Subject Classification (2000): Primary 60G60, 60H10, 60J60, 60K35, 82C22, $82 \mathrm{C} 31$ 
Such systems restricted to a finite time-interval (say [0, 1]), with $\mathbf{b}(\omega)=b(\omega(0))$ (Markovian drift), and when $b$ is the gradient of a smooth Hamilton function (see Section 6), were described as lattice Gibbs states on $\mathcal{C}([0,1], \mathbb{R})^{\mathbb{Z}^{d}}$ first by Deuschel in $[6,7]$ and later in [2].

Our aim is to deal with possibly non-Markovian and non-gradient systems in an infinite time-interval by using the concept of space-time Gibbs state introduced in [19]: we describe weak solutions of (1.1) as space-time Gibbs states on $\Omega$, and we give a variational characterization of them.

To be more precise, let $Q \in \mathcal{P}_{S}(\Omega)$ be a space-time invariant probability measure on $(\Omega, \mathcal{F})$, and $\mathbf{b}$ be a given function as above. Moreover we denote by $P$ the reference measure in $\mathcal{P}_{S}(\Omega)$, law of the stationary solution of equation (1.1) with $\mathbf{b} \equiv 0$. - The assumptions that will be given (see Section 4) on $\varphi$ will guarantee existence and uniqueness of such measure, as well as good ergodic properties -. Under the integrability condition

$$
\mathcal{H}(Q)<+\infty
$$

where $\mathcal{H}$ denotes the specific entropy of $Q$ with respect to $P$ (see Section 4), our main result is the equivalence of the following assertions:

[A] $Q$ is a stationary weak solution of the s.d.e. (1.1).

[B] $Q$ is a space-time invariant Gibbs state for a specification which is built on an Hamiltonian functional $H$, that is explicitely given in terms of $\mathbf{b}$. As customary in Equilibrium Statistical Models, this specification if defined as a perturbation of a reference specification, which in this model consists of stochastic bridges derived from $P$.

[C] $Q$ minimizes the free energy $\mathcal{H}^{\mathbf{b}}$, difference of the specific entropy and the specific energy, defined on $\mathcal{P}_{S}(\Omega)$ by

$$
\begin{aligned}
\mathcal{H}^{\mathbf{b}}\left(Q^{\prime}\right)= & \mathcal{H}\left(Q^{\prime}\right)-E_{Q^{\prime}}\left(\int_{0}^{1} \mathbf{b}\left(\theta_{0, t} \omega\right) d \omega_{0}(t)\right) \\
& +\frac{1}{2} E_{Q^{\prime}}\left(\mathbf{b}^{2}(\omega)-\mathbf{b}(\omega) \varphi^{\prime}\left(\omega_{0}(0)\right)\right)
\end{aligned}
$$

Assertion $[\mathrm{C}]$ can be stated in the following way:

$$
\begin{aligned}
0=\mathcal{H}^{\mathbf{b}}(Q)= & \inf _{\substack{Q^{\prime} \in \mathcal{P}_{s}(\Omega) \\
\mathcal{H}\left(Q^{\prime}\right)<+\infty}}\left[\mathcal{H}\left(Q^{\prime}\right)-E_{Q^{\prime}}\left(\int_{0}^{1} \mathbf{b}\left(\theta_{0, t} \omega\right) d \omega_{0}(t)\right)\right. \\
& \left.+\frac{1}{2} E_{Q^{\prime}}\left(\mathbf{b}^{2}(\omega)-\mathbf{b}(\omega) \varphi^{\prime}\left(\omega_{0}(0)\right)\right)\right] .
\end{aligned}
$$

If we consider the specific energy, i.e. the second part of $\mathcal{H}^{\mathbf{b}}$, we see that it includes a stochastic integral. Hamiltonians of this form appeared in Mathematical Physics in the context of diamagnetic current [17].

Gibbs fields on $\mathbb{R}^{\mathbb{Z}^{d}}$ have been considered by several authors from the variational principle point of view (see e.g. Föllmer [8], Lebowitz and Presutti [16], Künsch [15]), the difficulty coming from unboundedness of the spin space. On the other 
hand, Gibbs fields on the trajectory space $\mathcal{C}(\mathbb{R}, \mathbb{R})$ were introduced in the context of Euclidean quantum field theory as quasi-invariant measures (see Courrège and Renouard [1], Royer and Yor [26] and references therein). The variational approach of such Gibbs fields was first considered by Roelly and Zessin [24] for the law of a Brownian diffusion with values in the torus. More recently Osada and Spohn [21] used D-L-R approach for constructing a class of Gibbsian non Markovian real valued stochastic processes. One of the difficulties in dealing with Gibbs fields on path spaces, comes from the fact that disjoint time regions are not independent under the reference measure. In this paper, following the notions introduced by Minlos, Roelly and Zessin in [19], we deal with Gibbs fields on $\mathcal{C}(\mathbb{R}, \mathbb{R})^{\mathbb{Z}^{d}}$, that is parametrized by space and time $\mathbb{Z}^{d} \times \mathbb{R}$, so that both difficulties above have to be overcome. In particular, the dependence of a stochastic bridge on boundary conditions becomes essential, and methods from stochastic analysis play a key role.

We finally make some comments on the usefulness of the results proved in this paper. First of all we have not assumed Markovianity of the system, i.e. $\mathbf{b}(\omega)=$ $b(\omega(0))$. Moreover, even in the Markovian case, not having assumed any smoothness on the drift $\mathbf{b}$, it is not known whether the s.d.e. (1.1) admits any (weak or strong) solution, or whether among the solutions there is one that is time stationary. Our results suggest two approaches to the existence of stationary solutions.

- Stationary solutions may be constructed as space-time Gibbs states, e.g. via cluster expansion. Results concerning space-time cluster expansion for some models can be found e.g. in [19] and [20]. Our results guarantee that states constructed in that way are indeed weak stationary solutions of the s.d.e.. Indeed, we show in [5] that a stationary solution of (1.1) can be constructed by cluster expansion, provided $\|\mathbf{b}\|_{\infty}$ is sufficiently small, but with no Markovianity or regularity conditions.

- Stationary solutions may be obtained by exploiting the variational principle, i.e. by showing that the free energy $\mathcal{H}^{\mathbf{b}}$ attains the value 0 . This is the case if $\mathcal{H}^{\mathbf{b}}$ is the (good) rate function for a Large Deviation Principle. This would require some refinements of the results in [3, 4] concerning space-time large deviations.

The paper is organized in the following sections.

1. Introduction.

2. Gibbs specifications and space-time Gibbs states.

3. Infinite-dimensional diffusions are Gibbs states.

4. Weak variational principle.

5. The minima of the free energy are diffusions.

6. The case of Markovian gradient systems.

\section{Gibbs specifications and space-time Gibbs states}

First of all we introduce our one dimensional reference process, which is the timestationary solution of the scalar differential equation

$$
d x(t)=-\frac{1}{2} \varphi^{\prime}(x(t)) d t+d w(t)
$$


where $w$ is a real valued Brownian motion and the self potential $\varphi$ is a $\mathcal{C}^{2}(\mathbb{R}, \mathbb{R})$ function satisfying the following properties :

$$
\begin{gathered}
\lim _{|x| \rightarrow+\infty} \varphi(x)=+\infty, \text { and } \exists C_{0} \in \mathbb{R} \text { such that } \tilde{\varphi}=: \varphi^{\prime \prime}-\frac{1}{2}\left(\varphi^{\prime}\right)^{2} \leq C_{0} \\
e^{-\varphi} \in L^{1}(\mathbb{R}) .
\end{gathered}
$$

Property (2.2) guarantees that, for any given initial condition, a unique non-exploding strong solution of (2.1) exists (see Theorem 2.2.19 in [25]). Property (2.3) insures that the measure $e^{-\varphi} d x$, which is invariant, is normalizable. We let $W$ denote the law on $\mathcal{C}(\mathbb{R}, \mathbb{R})$ of the stationary solution of $(2.1)$, and let $\mu(d x)=$ $e^{-\varphi(x)} d x / \int e^{-\varphi(y)} d y$ denote the unique invariant Probability measure associated to $(2.1)$.

Let $\Omega=\mathcal{C}(\mathbb{R}, \mathbb{R})^{\mathbb{Z}^{d}}$ be the canonical configuration space, and $\mathcal{F}$ be the canonical $\sigma$-field. With $\mathcal{P}(\Omega)$ we denote the space of probability measures on $\Omega$, and $\mathcal{P}_{S}(\Omega)$ is the subset of $\mathcal{P}(\Omega)$ containing the probabilities that are invariant for the

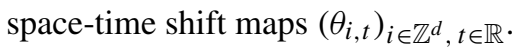

In what follows we let $P$ be the law of the reference non-interacting infinite system, i.e.

$$
P=\otimes_{\mathbb{Z}^{d}} W \in \mathcal{P}_{S}(\Omega) .
$$

The main object of this paper is an infinite-dimensional diffusion that is obtained by perturbing through an interaction a system of infinitely many independent particles each evolving with dynamics given by (2.1): we fix a finite subset $\Lambda_{0} \subset \mathbb{Z}^{d}$, and assume we are given a measurable bounded $\Lambda_{0}$-local function $\mathbf{b}(\omega)=\mathbf{b}\left(\omega_{\Lambda_{0}}\right)$ on $\mathcal{C}((-\infty, 0], \mathbb{R})^{\Lambda_{0}}$, where this path space is provided with the topology of uniform convergence on compact subsets of $\mathbb{R}^{-}$, and the corresponding Borel $\sigma$-field. We consider the associated stochastic differential system

$$
d X_{i}(t)=\left(-\frac{1}{2} \varphi^{\prime}\left(X_{i}(t)\right)+\mathbf{b}\left(\theta_{i, t} X\right)\right) d t+d B_{i}(t), \quad i \in \mathbb{Z}^{d}, t \in \mathbb{R}^{+} .
$$

Remark that in time-stationary situation, we can also consider the above system for time $t \in \mathbb{R}$. We need different filtrations on the space-time structure. Let $\mathcal{V}$ be the set of space-time volumes $V$ having the form $V=\Lambda \times I$ where $\Lambda \subset \mathbb{Z}^{d}$ finite, and $I=] a_{1}, a_{2}$ [ is a bounded open interval. For a space-volume $\Lambda \subset \mathbb{Z}^{d}$ we define its enlargement $\Lambda^{+}$by

$$
\Lambda^{+}=\left\{i \in \mathbb{Z}^{d}:\left(\Lambda_{0}+i\right) \cap \Lambda \neq \emptyset\right\} .
$$

Letting $\Lambda^{++}=\left(\Lambda^{+}\right)^{+}$, we define the boundary

$$
\partial \Lambda=\Lambda^{++} \backslash \Lambda \text {. }
$$

For a time-volume $I=] a_{1}, a_{2}\left[\subset \mathbb{R}\right.$ we define its enlargement $I^{+}$by

$$
\left.\left.I^{+}=\right]-\infty, a_{2}\right] \text {. }
$$


For $V=\Lambda \times I \in \mathcal{V}$ the forward $\sigma$-field $\mathcal{F}_{V}$ is defined by

$$
\mathcal{F}_{V}=\sigma\left\{\omega_{i}(t): i \in \Lambda^{++}, t \in I^{+}\right\},
$$

the backward $\sigma$-field $\hat{\mathcal{F}}_{V}$ by

$$
\hat{\mathcal{F}}_{V}=\sigma\left\{\omega_{i}(t):(i, t) \notin V\right\}
$$

and the boundary $\sigma$-field $\partial \mathcal{F}_{V}$ by

$$
\partial \mathcal{F}_{V}=\mathcal{F}_{V} \cap \hat{\mathcal{F}}_{V}
$$

We need now a reference specification, denoted by $\Pi^{0}$. It is a kernel based on $P$, and determined by

$$
\Pi_{V}^{0}(A)=P\left(A \mid \hat{\mathcal{F}}_{V}\right) \quad P-\text { a.s. }
$$

for all $V \in \mathcal{V}$, and $A \in \mathcal{F}_{V}$. It is easy to see that, for $\left.V=\Lambda \times\right] a_{1}, a_{2}\left[, \Pi_{V}^{0}\right.$ is given by

$$
\Pi_{V}^{0}\left(\omega, d \omega^{\prime}\right)=\otimes_{i \in \partial \Lambda} \delta_{\omega(i)}\left(d \omega_{i}^{\prime}\right) \otimes \otimes_{i \in \Lambda} W_{a_{1}, \omega_{i}\left(a_{1}\right)}^{a_{2}, \omega_{i}\left(a_{2}\right)}\left(d \omega_{i}^{\prime}\right)
$$

where $W_{a_{1}, x}^{a_{2}, y}$ is the law of the stochastic bridge obtained by conditioning $W$ to be $x$ at time $a_{1}$ and $y$ at time $a_{2}$. That $\Pi^{0}$ is a space-time specification in the Gibbsian sense has been proved in [19], Example 2, Section 1.4.2.

We now introduce the interaction between the spins through a potential $\Phi=$ $\left(\Phi_{V}\right)_{V \in \mathcal{V}}$ which is defined on a subset $\Omega^{\prime} \subset \Omega$ as follows:

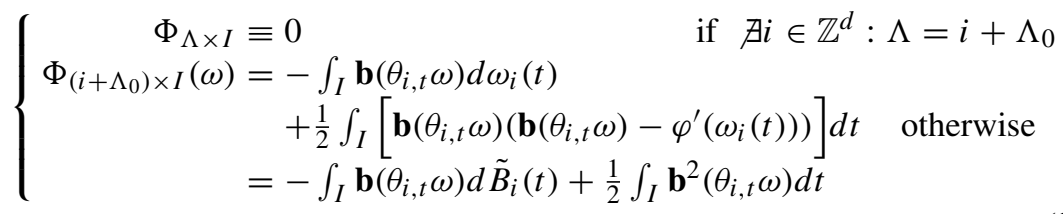

where

$$
\left.\tilde{B}_{i}(t)=\omega_{i}(t)+\frac{1}{2} \int_{a_{1}}^{t} \varphi^{\prime}\left(\omega_{i}(s)\right) d s, t \in\right] a_{1}, a_{2}[.
$$

Note that this potential is not defined a priori on the whole $\Omega$, but only for $\omega \in \Omega^{\prime}$ for which the stochastic integral $\int_{I} \mathbf{b}\left(\theta_{i, t} \omega\right) d \omega_{i}(t)$ makes sense.

Note also that $\tilde{B}_{i}\left(a_{1}+.\right)-\tilde{B}_{i}\left(a_{1}\right)$ 's are independent Brownian motions under $P$.

The associated Hamiltonian is defined for $V=\Lambda \times I$ by

$$
\begin{aligned}
H_{V}(\omega) & =\sum_{\Lambda^{\prime} \cap \Lambda \neq \emptyset} \Phi_{\Lambda^{\prime} \times I}(\omega) \\
& =-\sum_{i \in \Lambda^{+}}\left[\int_{I} \mathbf{b}\left(\theta_{i, t} \omega\right) d \tilde{B}_{i}(t)-\frac{1}{2} \int_{I} \mathbf{b}^{2}\left(\theta_{i, t} \omega\right) d t\right], \quad \omega \in \Omega^{\prime} .
\end{aligned}
$$


We observe that $\Phi$ and $H$ are space-time translation invariant, and that $H_{V}$ is $\mathcal{F}_{V}$-measurable. Moreover $\Phi$ has finite range equal to the diameter of $\Lambda_{0}$.

By means of $\Pi^{0}$, the reference specification, and $H$, the space-time Hamiltonian defined in (2.8), we define for $V \in \mathcal{V}, \omega \in \Omega$ the specification $\Pi_{V}^{H}(\omega,$.$) as$ the following probability measure on $\Omega^{\prime}$

$$
\Pi_{V}^{H}\left(\omega, d \omega^{\prime}\right)= \begin{cases}\frac{1}{Z_{V}^{H}(\omega)} \exp \left(-H_{V}\left(\omega^{\prime}\right)\right) \Pi_{V}^{0}\left(\omega, d \omega^{\prime}\right) & \text { if } 0<Z_{V}^{H}(\omega)<+\infty \\ 0 & \text { otherwise, }\end{cases}
$$

where

$$
Z_{V}^{H}(\omega)=\int_{\Omega^{\prime}} \exp \left(-H_{V}\left(\omega^{\prime}\right)\right) \Pi_{V}^{0}\left(\omega, d \omega^{\prime}\right)
$$

is the $\left(\partial \mathcal{F}_{V}\right.$-measurable) normalization factor.

Remark 1. 1. Note that $\Phi_{\left(i+\Lambda_{0}\right) \times I} \in L^{2}(P)$, and therefore is finite $P$-almost surely. We make the convention that it is always chosen in such a way that it does not assume the value $-\infty$.

2. The fundamental property

$$
E_{P}\left[\exp \left(-H_{V}\right)\right]=1 \quad \forall V \in \mathcal{V}
$$

holds. It follows from the fact that, for each $\Lambda \subset \mathbb{Z}^{d}$ finite and $a_{1} \in \mathbb{R}$ the process $\left(\exp \left(-H_{\Lambda \times] a_{1}, a_{2}[}\right)\right)_{a_{2}>a_{1}}$ is a $P$-martingale for the filtration $\left(\mathcal{F}_{\Lambda \times] a_{1}, a_{2}[}\right)_{a_{2}>a_{1}}$.

3. The fact that $\Pi^{H}$ is a specification comes from a general result of Preston [22].

Definition 1. A probability measure $Q$ on $\Omega$ is said to be a space-time Gibbs state with specification $\Pi^{H}$ if there exists a subset $\Omega^{\prime} \subset \Omega$ such that $Q\left(\Omega^{\prime}\right)=1, H$ is well defined on $\Omega^{\prime}$ and, for all $V \in \mathcal{V}$ and $A \in \mathcal{F}_{V} \cap \Omega^{\prime}$

$$
Q\left(A \mid \hat{\mathcal{F}}_{V}\right)=\Pi_{V}^{H}(A) Q-\text { a.s. }
$$

The set of space-time Gibbs states for $\Pi^{H}$ will be denoted by either $\mathcal{G}\left(\Pi^{H}\right)$ or $\mathcal{G}\left(H, \Pi^{0}\right)$.

Moreover we let $\mathcal{G}_{s}\left(\Pi^{H}\right)$ denote the set of space-time invariant Gibbs states, i.e.

$$
\mathcal{G}_{S}\left(\Pi^{H}\right)=\mathcal{G}\left(\Pi^{H}\right) \cap \mathcal{P}_{s}(\Omega) .
$$

\section{Infinite-dimensional diffusions are Gibbs states}

We recall that a weak solution of the s.d.e. (2.4) is a probability measure $Q$ on $\Omega$ such that the scalar processes

$$
\left(X_{i}(\cdot)-\int_{0}^{\cdot}\left(-\frac{1}{2} \varphi^{\prime}\left(X_{i}(s)\right)+\mathbf{b}\left(\theta_{i, s} X\right)\right) d s\right)_{i \in \mathbb{Z}^{d}}
$$

are $Q$-independent Brownian motions, where $X$ is the canonical process. 
Proposition 1. Let $Q \in \mathcal{P}_{S}(\Omega)$ be a weak solution of the s.d.e. (2.4). Then $Q \in$ $\mathcal{G}_{s}\left(\Pi^{H}\right)$ where the Hamiltonian $H$ is defined in (2.8).

Proposition 1 is a direct consequence of the following two lemmas.

Lemma 1. Let $Q^{1}, Q^{2}$ be two mutually absolutely continuous probability measures on a measurable space $\left(\Omega, \mathcal{F}^{\prime}\right)$, and let $\mathcal{F}$ be a sub- $\sigma$-field of $\mathcal{F}^{\prime}$. We denote by $Q_{\mathcal{F}}^{1}\left(\right.$ resp. $\left.Q_{\mathcal{F}}^{2}\right)$ the regular version of $Q^{1}(\cdot \mid \mathcal{F})\left(\right.$ resp. $\left.Q^{2}(\cdot \mid \mathcal{F})\right)$. Then, $Q^{1}$-a.s., $Q_{\mathcal{F}}^{1}$ and $Q_{\mathcal{F}}^{2}$ are mutually absolutely continuous and, denoting $M=d Q^{1} / d Q^{2}$, we have

$$
\frac{d Q_{\mathcal{F}}^{1}}{d Q_{\mathcal{F}}^{2}}=M Q_{\mathcal{F}}^{2}\left(M^{-1}\right)
$$

Proof. Let $f$ and $g$ be respectively $\mathcal{F}^{\prime}$-measurable and $\mathcal{F}$-measurable bounded functions. By definition of conditional expectation

$$
Q^{2}(f g)=Q^{2}\left(g Q_{\mathcal{F}}^{2}(f)\right) .
$$

On the other hand

$$
\begin{aligned}
Q^{2}(f g) & =Q^{1}(M f g)=Q^{1}\left(g Q_{\mathcal{F}}^{1}(M f)\right) \\
& =Q^{2}\left(M^{-1} g Q_{\mathcal{F}}^{1}(M f)\right)=Q^{2}\left(g Q_{\mathcal{F}}^{2}\left(M^{-1}\right) Q_{\mathcal{F}}^{1}(M f)\right)
\end{aligned}
$$

and the conclusion follows.

Lemma 2. Let $Q$ be a weak solution of (2.4) and let $I=] a_{1}, a_{2}[$. Define the $\sigma$-fields

$$
\mathcal{G}_{V}=\mathcal{G}_{\Lambda \times I}=\sigma\left\{\omega_{i}(t):\left(t \leq a_{1}, i \in \mathbb{Z}^{d}\right) \text { or }\left(t \leq a_{2}, i \notin \Lambda\right)\right\}
$$

and

$$
\mathcal{B}_{V}=\sigma\left\{\omega_{i}(t):(i, t) \in \Lambda \times \bar{I}\right\} .
$$

If $Q_{\mathcal{G}_{V}}$ and $P_{\mathcal{G}_{V}}$ are the regular versions of $Q\left(\cdot \mid \mathcal{G}_{V}\right)$ and $P\left(\cdot \mid \mathcal{G}_{V}\right)$, then

$$
\left.\frac{d Q_{\mathcal{G}_{V}}}{d P_{\mathcal{G}_{V}}}\right|_{\mathcal{B}_{V}}=\frac{1}{C_{V}(\omega)} \exp \left(-\sum_{i \in \Lambda^{+}}\left(-\int_{I} \mathbf{b}\left(\theta_{i, t} \omega\right) d \tilde{B}_{i}(t)+\frac{1}{2} \int_{I} \mathbf{b}^{2}\left(\theta_{i, t} \omega\right) d t\right)\right) .
$$

The normalization factor $C_{\Lambda \times I}(\omega)$ is measurable with respect to the $\sigma$-field

$$
\sigma\left\{\omega_{i}(t):\left(t \leq a_{1}, i \in \Lambda^{++}\right) \text {or }\left(t<a_{2}, i \in \partial \Lambda\right)\right\},
$$

thus, in particular, $C_{V}(\omega)$ is $\partial \mathcal{F}_{V}$-measurable.

Proof. Given the weak solution $Q$ of (2.4), define the measure $R$ on $\Omega$ by

$$
\frac{d R}{d Q}=\exp \left(\sum_{i \in \Lambda^{+}}\left(-\int_{I} \mathbf{b}\left(\theta_{i, t} \omega\right) d \tilde{B}_{i}(t)+\frac{1}{2} \int_{I} \mathbf{b}^{2}\left(\theta_{i, t} \omega\right) d t\right)\right) .
$$

By Girsanov Theorem it follows that $R$ is a probability measure : it is the weak solution of a similar equation to (2.4) but where the coordinates inside of $\Lambda^{+}$are simply independent diffusions evolving like (2.1). Then $\left.R_{\mathcal{G}_{V}}\right|_{\mathcal{B}_{V}}=P_{\mathcal{G}_{V}} \mid \mathcal{B}_{V}$. The conclusion follows by applying Lemma 1. 
Proof of Proposition 1. Observe that

$$
\hat{\mathcal{F}}_{V}=\mathcal{G}_{V} \vee \mathcal{G}_{V}^{\prime}
$$

where $\mathcal{G}_{V}$ is defined in Lemma 2, and

$$
\mathcal{G}_{V}^{\prime}=\sigma\left\{\omega_{i}\left(a_{2}\right): i \in \Lambda\right\}
$$

By combining Lemma 2 with Lemma 1 the conclusion follows.

Remark 2. In the literature there are strong regularity conditions on a Markovian drift to ensure existence (and uniqueness) of strong solutions to equation (2.4) (cf. e.g. [27], theorem 4.1). One advantage of our Gibbsian approach to infinite-dimensional diffusions, is that Gibbs fields can be constructed through perturbation methods (e.g. cluster expansion) without Markovianity and such regularity conditions $([5])$.

\section{Weak variational principle}

In this section we prove one direction of the space-time Gibbs variational principle, which is a partial converse of Proposition 1.

To simplify the notations, we introduce

$$
\mathcal{B}_{n}:=\mathcal{B}_{V_{n}}=\mathcal{B}_{\left.\Lambda_{n} \times\right] 0, n[}
$$

where $\Lambda_{n}=[0, n-1]^{d} \cap \mathbb{Z}^{d}$. We consider also

$$
\mathcal{B}^{-}=\sigma\left\{\omega_{i}(t):\left(t \leq 0, i \in \mathbb{Z}^{d}\right) \text { or }(0<t \leq 1 \text { and } i<0)\right\} .
$$

Here " $<"$ denotes the lexicographic order in $\mathbb{Z}^{d}$.

For $\mu, v$ probability measures on some measurable space $(E, \mathcal{E})$, we denote by $h(\mu \mid \nu)$ their relative entropy. Moreover $h_{\mathcal{G}}(\mu \mid \nu)$ is the relative entropy between their restriction $\left.\mu\right|_{\mathcal{G}}$ and $\left.\nu\right|_{\mathcal{G}}$ to a given sub- $\sigma$-field $\mathcal{G}$ of $\mathcal{E}$. As before, we denote by $\mu_{\mathcal{G}}$ the regular version of the conditional probability of $\mu$ w.r.t. $\mathcal{G}$.

We now give supplementary conditions on the self-potential $\varphi$, to ensure that the reference process $x(t)$ solution of (2.1) is sufficiently ergodic.

Assumption A. The self potential $\varphi$ satisfies either one of the following conditions:

A1.

$$
0<\liminf _{|x| \rightarrow+\infty} \varphi^{\prime \prime}(x) \leq \limsup _{|x| \rightarrow+\infty} \varphi^{\prime \prime}(x)<+\infty
$$

A2. Property (2.2) and

$$
0<\liminf _{|x| \rightarrow+\infty} \varphi^{\prime \prime}(x) \text { and } \int^{\infty} \frac{1}{\varphi^{\prime}(x)} d x<+\infty .
$$


The assumption $\lim \inf _{|x| \rightarrow+\infty} \varphi^{\prime \prime}(x)>0$ implies that the measure $e^{-\varphi(x)} d x$ has tails not bigger than Gaussian. The remaining parts of either Assumption A1 or Assumption A2 imply the following bounds on the transition density

$$
q_{t}(x, y)=\frac{p_{t}(x, y)}{e^{-\varphi(x)} / \int e^{-\varphi(y)} d y}
$$

with respect to the invariant probability measure $\mu(d x)$ for the diffusion (2.1), where $p_{t}(x, y)$ is the transition density from $y$ to $x$ with respect to the Lebesgue measure.

Lemma 3. a. Under Assumption A1 there is a constant $A>0$ such that for all $t \geq 1$ and $x, y \in \mathbb{R}$,

$$
e^{-A\left(x^{2}+y^{2}+1\right)} \leq q_{t}(x, y) \leq e^{A\left(x^{2}+y^{2}+1\right)} .
$$

b. Under Assumption A2 the stronger uniform bounds

$$
e^{-A} \leq q_{t}(x, y) \leq e^{A}
$$

hold for all $t \geq 1$ and $x, y \in \mathbb{R}$.

The proof of Lemma 3 will be given in the appendix.

A typical example of a potential satisfying A1 is a quadratic potential smoothly perturbed on a compact subset of $\mathbb{R}$; a typical example of a potential satisfying A2 is

$$
\varphi(x)=|x|^{s+2}, \quad \text { for some } s>0 .
$$

For $Q \in \mathcal{P}_{s}(\Omega)$ we define

$$
\mathcal{H}(Q)=Q\left(h_{\mathcal{B}_{1}}\left(Q_{\mathcal{B}^{-}} \mid W_{\omega_{0}(0)}\right)\right)
$$

where $W_{x}$ is the law of the solution of (2.1) with value $x \in \mathbb{R}$ at time 0 (note that $\left.W_{\omega_{0}(0)}=P_{\mathcal{B}^{-}} \mid \mathcal{B}_{1}\right)$. Furthermore, if $v$ is the law of $X_{0}(0)$ under $Q \in \mathcal{P}_{s}(\Omega)$, then the condition $\mathcal{H}(Q)<+\infty$ implies that

$$
\left.Q\right|_{\mathcal{B}_{1}} \ll W_{v}
$$

where $W_{v}(d \omega)=W_{x}(d \omega) \otimes v(d x)$ is the law of the solution of (2.1) with initial distribution $v$. This fact guarantees that every coordinate is a semimartingale under $Q$, and then that the stochastic integral under $Q$ makes sense :

$$
\mathcal{H}^{\mathbf{b}}(Q)= \begin{cases}\mathcal{H}(Q)-E_{Q}\left(\int_{0}^{1} \mathbf{b}\left(\theta_{0, t} \omega\right) d \omega_{0}(t)\right) & \\ +\frac{1}{2} E_{Q}\left(\mathbf{b}^{2}(\omega)-\mathbf{b}(\omega) \varphi^{\prime}\left(\omega_{0}(0)\right)\right) & \text { if } \mathcal{H}(Q)<+\infty \\ +\infty & \text { otherwise }\end{cases}
$$


We now prepare the proof of the variational principle. Let $Q \in \mathcal{G}_{s}\left(\Pi^{H}\right)$ satisfy $\mathcal{H}(Q)<+\infty$. Instead of $\Pi^{H}$ we also consider the specification $\Pi^{\tilde{H}}$, built on $\Pi^{0}$ and the following modified Hamiltonian

$$
\begin{aligned}
\tilde{H}_{V}(\omega)=- & \sum_{i \in \Lambda}\left(\int_{a_{1}+1}^{a_{2}-1} \mathbf{b}\left(\theta_{i, t} \omega\right) d \omega_{i}(t)\right. \\
& \left.-\frac{1}{2} \int_{a_{1}+1}^{a_{2}-1}\left(\mathbf{b}\left(\theta_{i, t} \omega\right)\left(\mathbf{b}\left(\theta_{i, t} \omega\right)-\varphi^{\prime}\left(\omega_{i}(t)\right)\right)\right) d t\right)
\end{aligned}
$$

for $V \in \mathcal{V}, V=\Lambda \times] a_{1}, a_{2}\left[\right.$ with $a_{1} \leq a_{2}-2$. We let $Z_{V}^{\tilde{H}}(\xi)$ denote the corresponding normalization

$$
Z_{V}^{\tilde{H}}(\xi)=\int_{\Omega} \exp \left(-\tilde{H}_{V}(\omega)\right) \Pi^{0}(\xi, d \omega) .
$$

We also introduce the following sequence of probability measures on $(\Omega, \mathcal{F})$, defined for $n \geq 2$,

$$
\Pi_{n, Q}^{\tilde{H}}\left(d \omega_{V_{n}}, d \xi_{V_{n}^{c}}\right)=\Pi_{V_{n}}^{\tilde{H}}(\xi, d \omega) Q(d \xi)
$$

The reason for using the modified Hamiltonian $\tilde{H}_{V}$ is that it is simpler to get estimates for $Z_{V}^{\tilde{H}}$ than for $Z_{V}^{H}$. Indeed, in computing $Z_{V}^{\tilde{H}}$ one needs only the restriction of $\Pi_{V}^{0}$ to the time interval $\left[a_{1}+1, a_{2}-1\right]$, and this restriction is very regular with respect to $P$.

In a first step we prove

Proposition 2. If $Q \in \mathcal{P}_{S}(\Omega)$ is such that $\mathcal{H}(Q)<+\infty$, then

$$
\lim _{n \rightarrow \infty} \frac{1}{\left|V_{n}\right|} h_{\mathcal{B}_{n}}\left(Q \mid \Pi_{n, Q}^{\tilde{H}}\right)=\mathcal{H}^{\mathbf{b}}(Q)
$$

where $\left|V_{n}\right|=n^{d+1}$ is the volume of the space-time region $V_{n}$.

As a second step we show

Proposition 3. If $Q \in \mathcal{G}_{S}\left(\Pi^{H}\right)$ is such that $\mathcal{H}(Q)<+\infty$, then

$$
\lim _{n \rightarrow \infty} \frac{1}{\left|V_{n}\right|} h_{\mathcal{B}_{n}}\left(Q \mid \Pi_{n, Q}^{\tilde{H}}\right)=0 .
$$

Thus we obtain, as the main result of this section, one direction of the Gibbs variational principle.

Theorem 1. If $Q \in \mathcal{G}_{s}\left(\Pi^{H}\right)$ is such that $\mathcal{H}(Q)<+\infty$, then $\mathcal{H}^{\mathbf{b}}(Q)=0$.

Remark 3. We make here some considerations of the finite entropy condition $\mathcal{H}(Q)$ $<+\infty$.

1. First of all, it implies that under $Q$ the canonical process is a semi-martingale, and then, the Hamiltonian $H$ is well defined $Q$-a.s. without any difficulty. 
2. Föllmer et al. introduced in [10], Definition 2.1, a locally finite entropy condition which can be stated as follows. Let $\hat{\mathcal{B}}^{-}$denote the $\sigma$-field

$$
\hat{\mathcal{B}}^{-}=\sigma\left\{\omega_{i}(t):\left(t \leq 0 ; i \in \mathbb{Z}^{d}\right) \text { or }(0<t \leq 1 \text { and } i \neq 0)\right\} .
$$

$Q$ satisfies the locally finite entropy condition if

$$
\hat{\mathcal{H}}(Q):=Q\left[h_{\mathcal{B}_{1}}\left(Q_{\hat{\mathcal{B}}^{-}} \mid W_{\omega_{0}(0)}\right)\right]<+\infty .
$$

Since $\mathcal{B}^{-} \subset \hat{\mathcal{B}}^{-}$, Jensen's inequality yields

$$
\hat{\mathcal{H}}(Q) \leq \mathcal{H}(Q) .
$$

Therefore our finite entropy condition implies the locally finite entropy condition of Föllmer.

3. Suppose that the drift $\mathbf{b}$ is Markovian $(\mathbf{b}(\omega)=b(\omega(0)))$ and of gradient type, i.e. there is a local $\mathcal{C}^{2}$-function $\psi$ such that the drift $b$ is given by

$$
b(x)=\frac{\partial}{\partial x_{0}} \sum_{i \in \mathbb{Z}^{d}} \psi\left(\theta_{i} x\right)
$$

where $\theta_{i} x_{j}=x_{i+j}$ is the space shift on $\mathbb{R}^{\mathbb{Z}^{d}}$ (as customary, the sum in (4.11) is formal, but its derivative is well defined). For this class of systems, that will be referred to as gradient systems, the condition $\mathcal{H}(Q)<+\infty$ holds true for all $Q \in \mathcal{G}_{S}\left(\Pi^{H}\right)$. This and other aspects of gradient systems will be the subject of Section 6.

Before giving the proof of Proposition 2, let us recall an important inequality satisfied by the relative entropy, which will be used at several places in the proof.

Lemma 4. For every pair of probability measures $(\mu, v)$ on a measurable space $(E, \mathcal{E})$, and for every measurable function $f$ such that $f^{-} \in L^{1}(v)$,

$$
\log \int_{E} e^{f} d \mu \geq \int_{E} f d v-h_{\mathcal{E}}(v \mid \mu) .
$$

Proof. When $f$ is bounded above, this is the usual entropy inequality. To obtain it for $f^{-} \in L^{1}(v)$, it is enough to construct bounded approximations and the inequality remains true for the limit.

In the proof of Proposition 2 we need two technical facts. The first is the representation of the functional $\mathcal{H}(Q)$ given by

$$
\mathcal{H}(Q)=\lim _{n \rightarrow \infty} \frac{1}{\left|V_{n}\right|} h_{\mathcal{B}_{n}}(Q \mid P) .
$$

which holds for $Q \in \mathcal{P}_{S}(\Omega)$ such that $\mathcal{H}(Q)<+\infty$. This is a version of McMillan Theorem. It can be proved as in Dai Pra [4], Proposition 4.1, and goes back to the work of Robinson and Ruelle [23]. The second technical fact we need is stated and proved in the following simple but key Lemma. 
Lemma 5. Under Assumption $\mathrm{A}$, if $Q \in \mathcal{P}_{S}(\Omega)$ is such that $\mathcal{H}(Q)<+\infty$, then

$$
\lim _{n \rightarrow+\infty} \frac{1}{\left|V_{n}\right|} h_{\mathcal{B}_{\Lambda_{n} \times\{0, n\}}}(Q \mid P)=0 .
$$

Proof. We first note that

$$
\begin{aligned}
& \left.\log \frac{d Q}{d P}\right|_{\mathcal{B}_{\Lambda_{n} \times\{0, n\}}} \\
& =\left.\log \frac{d Q_{\mathcal{B}_{\Lambda_{n} \times\{0\}}}}{d P_{\mathcal{B}_{\Lambda_{n} \times\{0\}}}}\right|_{\mathcal{B}_{\Lambda_{n} \times\{n\}}}+\left.\log \frac{d Q}{d P}\right|_{\mathcal{B}_{\Lambda_{n} \times\{0\}}} \\
& =\left.\log \frac{d Q_{\mathcal{B}_{\Lambda_{n} \times\{0\}}}}{d P}\right|_{\mathcal{B}_{\Lambda_{n} \times\{n\}}}+\left.\log \frac{d P}{d P_{\mathcal{B}_{\Lambda_{n} \times\{0\}}}}\right|_{\mathcal{B}_{\Lambda_{n} \times\{n\}}}+\left.\log \frac{d Q}{d P}\right|_{\mathcal{B}_{\Lambda_{n} \times\{0\}}} .
\end{aligned}
$$

Using the time stationarity of $Q$ and Lemma 4 applied to $\mu=\left.Q\right|_{\mathcal{B}_{\Lambda_{n} \times\{0\}}}$,

$$
\begin{aligned}
& v=\left.Q\right|_{\mathcal{B}_{\Lambda_{n} \times\{n\}}} \text { and } f=\left.\log \frac{d Q_{\mathcal{B}_{\Lambda_{n} \times\{0\}}}}{d P}\right|_{\mathcal{B}_{\Lambda_{n} \times\{n\}}} \text { we have } \\
& E_{Q}\left[\left.\log \frac{d Q_{\mathcal{B}_{\Lambda_{n} \times\{0\}}}}{d P}\right|_{\mathcal{B}_{\Lambda_{n} \times\{n\}}}\right] \leq E_{Q}\left[\left.\log \frac{d Q}{d P}\right|_{\mathcal{B}_{\Lambda_{n} \times\{0\}}}\right]=h_{\mathcal{B}_{\Lambda_{n} \times\{0\}}}(Q \mid P) .
\end{aligned}
$$

Moreover

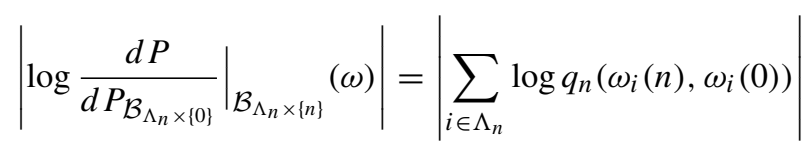

where $q_{t}(x, y)$ has been defined in Lemma 3 .

Now, under Assumption A2, $\left|\log q_{n}\right|$ is uniformly bounded so that, easily

$$
\lim _{n \rightarrow+\infty} \frac{1}{\left|V_{n}\right|} E_{Q}\left[\left.\log \frac{d P}{d P_{\mathcal{B}_{\Lambda_{n} \times\{0\}}}}\right|_{\mathcal{B}_{\Lambda_{n} \times\{n\}}}\right]=0 .
$$

On the other hand, under Assumption A1, thanks to Lemma 3, there is a positive constant $A$, independent on $n$, such that

$$
\left|\log q_{n}(x, y)\right| \leq A\left(1+x^{2}+y^{2}\right)
$$

Thus, by space-time shift invariance of $Q$

$$
\left|E_{Q}\left[\left.\log \frac{d P}{d P_{\mathcal{B}_{\Lambda_{n} \times\{0\}}}}\right|_{\mathcal{B}_{\Lambda_{n} \times\{n\}}}\right]\right| \leq n^{d}\left(2 E_{Q}\left[\omega_{0}^{2}(0)\right]+1\right) .
$$

Thus (4.16) is established under Assumption A1 if we show that $E_{Q}\left[\omega_{0}^{2}(0)\right]<+\infty$. To see this we use Lemma 4. For every $\epsilon>0$

$$
E_{Q}\left[\omega_{0}^{2}(0)\right] \leq \epsilon^{-1}\left[\log E_{P}\left(e^{\epsilon \omega_{0}^{2}(0)}\right)+h_{\mathcal{B}_{\{0\} \times\{0\}}}(Q \mid P)\right]
$$


that is finite for $\epsilon$ sufficiently small, since $\left.P\right|_{\mathcal{B}_{\{0\} \times\{0\}}}$ has Gaussian tails. Thus (4.16) is established under Assumption A1 too.

By (4.14), (4.15) and (4.16) we get

$$
h_{\mathcal{B}_{\Lambda_{n} \times\{0, n\}}}(Q \mid P) \leq 2 h_{\mathcal{B}_{\Lambda_{n} \times\{0\}}}(Q \mid P)+o\left(\left|V_{n}\right|\right) .
$$

The fact that

$$
h_{\mathcal{B}_{\Lambda_{n} \times\{0\}}}(Q \mid P)=o\left(\left|V_{n}\right|\right)
$$

is almost obvious. For instance, one can take in (4.13) the space-time volume $V_{n}=\Lambda_{n} \times[0, \sqrt{n}]$ and obtain

$$
\mathcal{H}(Q)=\lim _{n \rightarrow \infty} \frac{1}{n^{d+\frac{1}{2}}} h_{\mathcal{B}_{\Lambda_{n} \times[0, \sqrt{n}]}}(Q \mid P)
$$

which yields

$$
h_{\mathcal{B}_{\Lambda_{n} \times\{0\}}}(Q \mid P) \leq h_{\mathcal{B}_{\Lambda_{n} \times[0, \sqrt{n}]}}(Q \mid P)=o\left(n^{d+1}\right) .
$$

By (4.19) and (4.20) the conclusion follows.

Proof of Proposition 2. Taking into account the definition of $\mathcal{H}^{\mathbf{b}}$ and (4.13), all we have to show is

$$
\begin{aligned}
\lim _{n \rightarrow \infty} \frac{1}{\left|V_{n}\right|} E_{Q}\left(\left.\log \frac{d \Pi_{n, Q}^{\tilde{H}}}{d P}\right|_{\mathcal{B}_{n}}\right)= & E_{Q}\left(\int_{0}^{1} \mathbf{b}\left(\theta_{0, t} \omega\right) d \tilde{B}_{0}(t)\right. \\
& \left.-\frac{1}{2} \int_{0}^{1} \mathbf{b}^{2}\left(\theta_{0, t} \omega\right) d t\right) .
\end{aligned}
$$

Note that

$$
\left.\log \frac{d \Pi_{n, Q}^{\tilde{H}}}{d P}\right|_{\mathcal{B}_{n}}=\left.\log \frac{d \Pi_{V_{n}}^{\tilde{H}}\left(\omega_{V_{n}^{c}}, \cdot\right)}{d \Pi_{V_{n}}^{0}\left(\omega_{V_{n}^{c}}, \cdot\right)}\right|_{\mathcal{B}_{n}}+\left.\log \frac{d Q}{d P}\right|_{\mathcal{B}_{\Lambda_{n} \times\{0, n\}}} .
$$

By Lemma 5, (4.21) is established if we can show that

$$
\begin{aligned}
& \lim _{n \rightarrow+\infty} \frac{1}{\left|V_{n}\right|} E_{Q}\left[\left.\log \frac{d \Pi_{V_{n}}^{\tilde{H}}\left(\omega_{V_{n}^{c}}, \cdot\right)}{d \Pi_{V_{n}}^{0}\left(\omega_{V_{n}^{c}}, \cdot\right)}\right|_{\mathcal{B}_{n}}\right] \\
& \quad=E_{Q}\left(\int_{0}^{1} \mathbf{b}\left(\theta_{0, t} \omega\right) d \tilde{B}_{0}(t)-\frac{1}{2} \int_{0}^{1} \mathbf{b}^{2}\left(\theta_{0, t} \omega\right) d t\right) .
\end{aligned}
$$

To see this, observe that

$$
E_{Q}\left[\left.\log \frac{d \Pi_{V_{n}}^{\tilde{H}}\left(\omega_{V_{n}^{c}}, \cdot\right)}{d \Pi_{V_{n}}^{0}\left(\omega_{V_{n}^{c}}, \cdot\right)}\right|_{\mathcal{B}_{n}}\right]=-E_{Q}\left[\log Z_{V_{n}}^{\tilde{H}}\left(\omega_{V_{n}^{c}}\right)\right]-E_{Q}\left(\tilde{H}_{V_{n}}(\omega)\right) .
$$


Identity (4.22) now follows if we show that

$$
-\lim _{n \rightarrow+\infty} \frac{1}{\left|V_{n}\right|} E_{Q}\left(\tilde{H}_{V_{n}}(\omega)\right)=E_{Q}\left(\int_{0}^{1} \mathbf{b}\left(\theta_{0, t} \omega\right) d \tilde{B}_{0}(t)-\frac{1}{2} \int_{0}^{1} \mathbf{b}^{2}\left(\theta_{0, t} \omega\right) d t\right)
$$

and

$$
\lim _{n \rightarrow+\infty} \frac{1}{\left|V_{n}\right|} E_{Q}\left[\log Z_{V_{n}}^{\tilde{H}}\left(\omega_{V_{n}^{c}}\right)\right]=0
$$

Equality (4.24) follows readily by shift invariance of $Q$ and the definition of $\tilde{H}_{V_{n}}$, provided

$$
\int_{0}^{1} \mathbf{b}\left(\theta_{0, t} \omega\right) d \tilde{B}_{0}(t) \in L^{1}(Q) .
$$

To prove (4.26) we argue as follows. Due to the finite entropy condition, the canonical process under $Q$ has a semi-martingale decomposition as follows: there exists a $Q$-square integrable random variable $\beta$, measurable w.r.t. $\sigma\{\omega(t): t \leq 0\}$, such that

$$
\left(M_{i}(t):=\tilde{B}_{i}(t)-\int_{0}^{t} \beta\left(\theta_{i, s} \omega\right) d s\right)_{i \in \mathbb{Z}^{d}}
$$

are Q-independent Brownian motions. This fact can be proved by using Theorem 2.4 in [10] or [2] p.166, specialized to the translation invariant case. Indeed (see Remark 3), our entropy condition is stronger than the local entropy conditions appearing in [10].

Therefore

$$
\int_{0}^{1} \mathbf{b}\left(\theta_{0, t} \omega\right) d \tilde{B}_{0}(t)=\int_{0}^{1} \mathbf{b}\left(\theta_{0, t} \omega\right) \beta\left(\theta_{0, t} \omega\right) d t+\int_{0}^{1} \mathbf{b}\left(\theta_{0, t} \omega\right) d M_{0}(t)
$$

that easily implies (4.26) and thus (4.24).

We are left to show (4.25). First observe that (4.12)

$$
\begin{aligned}
\log Z_{V_{n}}^{\tilde{H}}\left(\omega_{V_{n}^{c}}\right) & =\log \int e^{-\tilde{H}_{V_{n}}} d \Pi_{V_{n}}^{0}\left(\omega_{V_{n}^{c}}, \cdot\right) \\
& =\left.\log \int e^{-\tilde{H}_{V_{n}}}\left(\left.\frac{d \Pi_{V_{n}}^{0}\left(\omega_{V_{n}^{c}}, \cdot\right)}{d P}\right|_{\mathcal{B}_{\Lambda_{n} \times[1, n-1]}}\right) d P\right|_{\mathcal{B}_{\Lambda_{n} \times[1, n-1]}} .
\end{aligned}
$$

Moreover

$$
\left.\frac{d \Pi_{V_{n}}^{0}\left(\omega_{V_{n}^{c}}, \cdot\right)}{d P}\right|_{\mathcal{B}_{\Lambda_{n} \times[1, n-1]}}\left(\omega_{V_{n}}\right)=\prod_{i \in \Lambda_{n}} \frac{q_{1}\left(\omega_{i}(1), \omega_{i}(0)\right) q_{1}\left(\omega_{i}(n), \omega_{i}(n-1)\right)}{q_{n}\left(\omega_{i}(n), \omega_{i}(0)\right)} .
$$


Under Assumption A2, each term of the r.h.s. of (4.29) is uniformly bounded, that immediately implies (4.25) after having observed that, by Girsanov formula,

$$
\int e^{-\tilde{H}_{V_{n}}} d P=1 .
$$

Under Assumption A1, we use the bound (4.17) on the transition density $q_{t}(x, y)$, and we get

$$
\begin{aligned}
& \left.\frac{d \Pi_{V_{n}}^{0}\left(\omega_{V_{n}^{c}}, \cdot\right)}{d P}\right|_{\mathcal{B}_{\Lambda_{n} \times[1, n-1]}} \\
& \geq \exp \left[-A^{\prime} \sum_{i \in \Lambda_{n}}\left(\omega_{i}^{2}(0)+\omega_{i}^{2}(1)+\omega_{i}^{2}(n-1)+\omega_{i}^{2}(n)+1\right)\right] .
\end{aligned}
$$

Thus, letting $d Q_{n}^{\omega_{V_{n}^{c}}}=\left.e^{-\tilde{H}_{V_{n}}\left(\omega_{V_{n}^{c}} \cdot\right)} d P\right|_{\mathcal{B}_{\Lambda_{n} \times[1, n-1]}}$, by Jensen's inequality

$$
\begin{aligned}
& \log Z_{V_{n}}^{\tilde{H}}\left(\omega_{V_{n}^{c}}\right) \\
& \geq \log \int \exp \left[-A^{\prime} \sum_{i \in \Lambda_{n}}\left(\omega_{i}^{2}(0)+\omega_{i}^{2}(1)+\omega_{i}^{2}(n-1)+\omega_{i}^{2}(n)+1\right)\right] d Q_{n}^{\omega_{V_{n}^{c}}} \\
& \geq-A^{\prime} \sum_{i \in \Lambda_{n}}\left\{E_{\left.Q_{n}{ }^{\omega_{V_{n}^{c}}}\left[\omega_{i}^{2}(1)+\omega_{i}^{2}(n-1)\right]+1+\omega_{i}^{2}(0)+\omega_{i}^{2}(n)\right\}}\right.
\end{aligned}
$$

Thus, the inequality

$$
\liminf _{n} \frac{1}{\left|V_{n}\right|} E_{Q}\left[\log Z_{V_{n}}^{\tilde{H}}\left(\omega_{V_{n}^{c}}\right)\right] \geq 0
$$

follows if we show that

$$
\sup _{n} \sup _{i \in \Lambda_{n}} \sup _{\omega_{n}^{c}} E{ }_{Q_{n}}^{\omega_{V_{n}^{c}}}\left[\omega_{i}^{2}(n-1)\right]<+\infty .
$$

To see (4.31), observe that, under $Q_{n}^{\omega} V_{n}^{c}$, the canonical process satisfies the s.d.e., for $t \in[1, n-1]$

$$
d \omega_{i}(t)=\left[-c \omega_{i}(t)+\mathbf{b}\left(\theta_{i, t} \omega\right)\right] d t+d N_{i}(t)
$$

where $\left(\omega_{i}(1)\right)_{i \in \Lambda_{n}}$ are i.i.d. and distributed according to the invariant measure of (2.1), and $\left(N_{i}\right)_{i \in \Lambda_{n}}$ are independent Brownian motions under $Q_{n}^{\omega_{V_{n}^{c}}}$. Boundedness of $\mathbf{b}(\cdot)$ and an elementary application of Ito's rule yield (4.31).

We are now left to show

$$
\limsup _{n} \frac{1}{\left|V_{n}\right|} E_{Q}\left[\log Z_{V_{n}}^{\tilde{H}}\left(\omega_{V_{n}^{c}}\right)\right] \leq 0
$$


under Assumption A1. We first claim that

$$
\left(\log Z_{V_{n}}^{\tilde{H}}\right)^{-} \in L^{1}(Q)
$$

This follows easily from (4.30), (4.31) and the fact that, as shown above, $E_{Q}\left(\omega_{0}^{2}(0)\right)$ $<+\infty$ for $Q$ satisfying $\mathcal{H}(Q)<+\infty$. By (4.33), we are allowed to use Lemma 4 and obtain

$$
E_{Q}\left[\log Z_{V_{n}}^{\tilde{H}}\right] \leq \log E_{P}\left(Z_{V_{n}}^{\tilde{H}}\right)+h_{\mathcal{B}_{\Lambda_{n} \times\{0, n\}}}(Q \mid P) .
$$

Using this inequality together with Lemma 5 and the fact that $E_{P}\left(Z_{V_{n}}^{\tilde{H}}\right)=1,(4.32)$ follows.

Proof of Proposition 3. Let $Q \in \mathcal{G}_{S}\left(\Pi^{H}\right)$ satisfy $\mathcal{H}(Q)<+\infty$. The following argument goes back to Preston [22], Lemma 7.7. First note the identity

$$
\left.\frac{d Q}{d \Pi_{n, Q}^{\tilde{H}}}\right|_{\mathcal{B}_{n}}\left(\omega_{V_{n}}, \xi_{V_{n}^{c}}\right)=\left.\frac{\Pi_{V_{n}}^{H}(\xi, d \omega)}{\Pi_{V_{n}}^{\tilde{H}}(\xi, d \omega)}\right|_{\mathcal{B}_{n}} .
$$

This follows from definition of $\Pi_{n, Q}^{\tilde{H}}$ and the Gibbs property of $Q$. Therefore

$$
\begin{aligned}
\log & \left.\frac{d Q}{d \Pi_{n, Q}^{\tilde{H}}}\right|_{\mathcal{B}_{n}}\left(\omega_{V_{n}}, \xi_{V_{n}^{c}}\right) \\
= & \sum_{i \in \Lambda_{n}^{+}}\left(\int_{0}^{n} \mathbf{b}\left(\theta_{i, t} \omega\right) d \tilde{B}_{i}(t)-\frac{1}{2} \int_{0}^{n} \mathbf{b}^{2}\left(\theta_{i, t} \omega\right) d t\right) \\
& -\sum_{i \in \Lambda_{n}}\left(\int_{1}^{n-1} \mathbf{b}\left(\theta_{i, t} \omega\right) d \tilde{B}_{i}(t)-\frac{1}{2} \int_{1}^{n-1} \mathbf{b}^{2}\left(\theta_{i, t} \omega\right) d t\right) \\
& -\log Z_{V_{n}}^{H}\left(\xi_{V_{n}^{c}}\right)+\log Z_{V_{n}}\left(\xi_{V_{n}^{c}}\right) .
\end{aligned}
$$

We now discuss separately the behavior of each term in (4.35). As in Proposition 2

$$
\lim _{n \rightarrow \infty} \frac{1}{\left|V_{n}\right|} E_{Q}\left[\log Z_{V_{n}}^{\tilde{H}}\left(\xi_{V_{n}^{c}}\right)\right]=0
$$

We observe also that the semimartingale argument used in Proposition 2 to prove (4.26), applies here as well to show that the integral with respect to $Q$ of the difference of the first two terms in (4.35) multiplied by $1 /\left|V_{n}\right|$ goes to zero. The proof is therefore completed if we can show that

$$
\liminf _{n \rightarrow \infty} \frac{1}{\left|V_{n}\right|} E_{Q}\left(\log Z_{V_{n}}^{H}\left(\xi_{V_{n}^{c}}\right)\right) \geq 0
$$


Using (4.12) we obtain

$$
\begin{aligned}
\log Z_{V_{n}}^{H}\left(\xi V_{n}^{c}\right) & \log \int \Pi_{V_{n}}^{0}(\xi, d \omega) e^{-H_{V_{n}}(\omega)} \\
\geq & -\int \Pi_{V_{n}}^{\tilde{H}}(\xi, d \omega) H_{V_{n}}(\omega)-\int \Pi_{V_{n}}^{\tilde{H}}(\xi, d \omega) \log \frac{\Pi_{V_{n}}^{\tilde{H}}(\xi, d \omega)}{\Pi_{V_{n}}^{0}(\xi, d \omega)} \\
= & \int \Pi_{V_{n}}^{\tilde{H}}(\xi, d \omega)\left[\sum_{i \in \Lambda_{n}^{+}}\left(\int_{0}^{n} \mathbf{b}\left(\theta_{i, t} \omega\right) d \tilde{B}_{i}(t)-\frac{1}{2} \int_{0}^{n} \mathbf{b}^{2}\left(\theta_{i, t} \omega\right) d t\right)\right. \\
& \left.-\sum_{i \in \Lambda_{n}}\left(\int_{1}^{n-1} \mathbf{b}\left(\theta_{i, t} \omega\right) d \tilde{B}_{i}(t)-\frac{1}{2} \int_{1}^{n-1} \mathbf{b}^{2}\left(\theta_{i, t} \omega\right) d t\right)\right]+\log Z_{V_{n}}^{\tilde{H}}\left(\xi_{V_{n}^{c}}\right) \\
= & \int \Pi_{V_{n}}^{\tilde{H}}(\xi, d \omega)\left[\sum_{i \in \Lambda_{n}^{+} \backslash \Lambda_{n}}\left(\int_{0}^{n} \mathbf{b}\left(\theta_{i, t} \omega\right) d \tilde{B}_{i}(t)-\frac{1}{2} \int_{0}^{n} \mathbf{b}^{2}\left(\theta_{i, t} \omega\right) d t\right)\right. \\
& \left.+\sum_{i \in \Lambda_{n}}\left(\int_{[0,1] \cup[n-1, n]} \mathbf{b}\left(\theta_{i, t} \omega\right) d \tilde{B}_{i}(t)-\frac{1}{2} \int_{[0,1] \cup[n-1, n]} \mathbf{b}^{2}\left(\theta_{i, t} \omega\right) d t\right)\right] \\
& +\log Z_{V_{n}}^{\tilde{H}}\left(\xi \xi_{V_{n}^{c}}\right) .
\end{aligned}
$$

The proof is complete if we can find a constant $C>0$ such that, for $i \in \Lambda_{n}^{+} \backslash \Lambda_{n}$

$$
\left|\int Q(d \xi) \Pi_{V_{n}}^{\tilde{H}}(\xi, d \omega) \int_{0}^{n} \mathbf{b}\left(\theta_{i, t} \omega\right) d \tilde{B}_{i}(t)\right| \leq C n
$$

and, for $i \in \Lambda_{n}$,

$$
\left|\int Q(d \xi) \Pi_{V_{n}}^{\tilde{H}}(\xi, d \omega)\left[\int_{[0,1]} \mathbf{b}\left(\theta_{i, t} \omega\right) d \tilde{B}_{i}(t)+\int_{[n-1, n]} \mathbf{b}\left(\theta_{i, t} \omega\right) d \tilde{B}_{i}(t)\right]\right| \leq C .
$$

The proof of (4.38) follows easily after having observed that $\tilde{B}_{i}(t)-\int_{0}^{t} \beta\left(\theta_{s, i} \xi\right) d s$ is a Brownian motion under $Q$, with $\beta$ as in Proposition 2.

The proof of (4.39) is slightly harder, since we have to find a semimartingale representation of $\tilde{B}_{i}(t)$ under $\Pi_{V_{n}}^{\tilde{H}}$. Consider first the part of (4.39) with integral on the time interval $[0,1]$. By definition of $\Pi_{V_{n}}^{\tilde{H}}$, in $[0,1]$ the law of $\tilde{B}_{i}(t)$ under $\Pi_{V_{n}}^{\tilde{H}}$ coincides with the law of the solution of $(2.1)$ conditioned to hold $\xi_{i}(0)$ at time 0 and $\xi_{i}(n)$ at time $n$. It is well known [12] that the drift of such process is given by

$$
-\frac{1}{2} \varphi^{\prime}(x)+\frac{d}{d x} \log p_{n-t}\left(\xi_{i}(n), x\right),
$$

where, as above, $p_{t}(\cdot, \cdot)$ is the transition density of the solution of (2.1). Thus, defining

$$
\lambda_{n, \xi}(t, x)=\frac{d}{d x} \log p_{n-t}\left(\xi_{i}(n), x\right)
$$


the process

$$
\tilde{B}_{i}(t)-\int_{0}^{t} \lambda_{n, \xi}\left(s, \omega_{i}(s)\right) d s
$$

for $0 \leq t \leq 1$ is a Brownian motion under $\Pi_{V_{n}}^{\tilde{H}}$. Therefore

$$
\begin{aligned}
& \left|\int \Pi_{V_{n}}^{\tilde{H}}(\xi, d \omega) \int_{[0,1] \cup[n-1, n]} \mathbf{b}\left(\theta_{i, t} \omega\right) d \tilde{B}_{i}(t)\right| \\
& \quad=\left|\int \Pi_{V_{n}}^{\tilde{H}}(\xi, d \omega) \int_{[0,1] \cup[n-1, n]} \mathbf{b}\left(\theta_{i, t} \omega\right) \lambda_{n, \xi}\left(t, \omega_{i}(t)\right) d t\right| \\
& \quad \leq \sqrt{2}\|\mathbf{b}\|_{\infty}\left[\int \Pi_{V_{n}}^{\tilde{H}}(\xi, d \omega) \int_{[0,1] \cup[n-1, n]} \lambda_{n, \xi}^{2}\left(t, \omega_{i}(t)\right) d t\right]^{1 / 2} .
\end{aligned}
$$

Now note that, by Girsanov Theorem, the quantity

$$
\frac{1}{2} \int \Pi_{V_{n}}^{\tilde{H}}(\xi, d \omega) \int_{0}^{1} \lambda_{n, \xi}^{2}\left(t, \omega_{i}(t)\right) d t
$$

equals the relative entropy of the restriction to the time interval $[0,1]$ of the Stochastic Bridge $W_{0, \xi_{i}(0)}^{n, \xi_{i}(n)}$ and the measure $W_{0, \xi_{i}(0)}$. By using elementary properties of the relative entropy, the relative entropy above is equal to the relative entropy between the projections at time 1 of $W_{0, \xi_{i}(0)}^{n, \xi_{i}(n)}$ and $W_{0, \xi_{i}(0)}$, that are given respectively by

$$
\frac{p_{1}\left(x, \xi_{i}(0)\right) p_{n-1}\left(\xi_{i}(n), x\right)}{p_{n}\left(\xi_{i}(n), \xi_{i}(0)\right)} d x=\frac{q_{1}\left(x, \xi_{i}(0)\right) q_{n-1}\left(\xi_{i}(n), x\right)}{q_{n}\left(\xi_{i}(n), \xi_{i}(0)\right)} \mu(d x)
$$

and

$$
p_{1}\left(x, \xi_{i}(0)\right) d x=q_{1}\left(x, \xi_{i}(0)\right) \mu(d x) .
$$

By using the same arguments in the proof of Proposition 2, based on the bounds for $q_{t}(x, y),(4.39)$ follows easily.

\section{The minima of the free energy are diffusions}

This section is devoted to the proof of the following result.

Theorem 2. Let $Q \in \mathcal{P}_{s}(\Omega)$ be such that $\mathcal{H}^{\mathbf{b}}(Q)=0$. Then $Q$ is a weak solution of the s.d.e. (2.4).

We begin with some technical lemmas. In what follows we let

$$
\begin{aligned}
\mathcal{G}_{n} & =\sigma\left\{\omega_{i}(t):(t \leq 0) \text { or }\left(t \leq 1, i \in \Lambda_{n}\right)\right\} \\
\mathcal{G}^{n} & =\sigma\left\{\omega_{i}(t):(t \leq 0) \text { or }\left(t \leq 1, i \notin \Lambda_{n}\right)\right\} \\
\mathcal{G}^{-} & =\sigma\left\{\omega_{i}(t): t \leq 0, i \in \mathbb{Z}^{d}\right\} .
\end{aligned}
$$


Lemma 6. Suppose $\mathcal{H}(Q)<\infty$. Then

$$
\mathcal{H}(Q)=\lim _{n \rightarrow \infty} \frac{1}{n^{d}} E_{Q}\left\{h_{\mathcal{G}_{n}}\left(Q_{\mathcal{G}^{-}} \mid P_{\mathcal{G}^{-}}\right)\right\} .
$$

The proof of Lemma 6 is standard, and is essentially equal to the proof of (4.13). From Lemma 6, by the same argument as in Lemma 5.5 of [4], we get the following result.

Lemma 7. Suppose $\mathcal{H}^{\mathbf{b}}(Q)=0$. Then

$$
\mathcal{H}(Q)=\lim _{n \rightarrow \infty} \frac{1}{n^{d}} E_{Q}\left(h_{\mathcal{G}_{n}}\left(Q_{\mathcal{G}^{n}} \mid P_{\mathcal{G}^{n}}\right)\right) .
$$

Lemma 8. Suppose $\mathcal{H}^{\mathbf{b}}(Q)=0$, and let $\beta$ be the $\mathcal{G}^{-}$-measurable random variable such that, for all $i \in \mathbb{Z}^{d}$

$$
M_{t}(i)=\tilde{B}_{i}(t)-\int_{0}^{t} \beta\left(\theta_{i, s} \omega\right) d s
$$

are independent Brownian motions under $Q$ (see (4.27)). Then

$$
\mathcal{H}(Q) \geq \frac{1}{2} E_{Q}\left(\beta^{2}\right)
$$

Proof. We sketch the proof of (5.2), although it is analogous to Lemma 5.7 in [4].

Define

$$
z_{t}^{n}=\exp \left[\sum_{i \in \Lambda_{n}}\left(\int_{0}^{t} \beta\left(\theta_{i, s} \omega\right) d \tilde{B}_{s}(i)-\frac{1}{2} \int_{0}^{t} \beta^{2}\left(\theta_{i, s} \omega\right) d s\right)\right] .
$$

By Girsanov Theorem, $z_{t}^{n}$ is a $P_{\mathcal{G}^{-}}$-local martingale. So let $\tau_{k}$ be an increasing sequence of stopping times, $\tau_{k} \rightarrow 1$, such that for all $k$ the process $z_{t \wedge \tau_{k}}^{n}$ is a martingale. We can therefore define the random probability measure $P_{\omega}^{n, k}$ by

$$
d P_{\omega}^{n, k}=z_{1 \wedge \tau_{k}}^{n} d P_{\mathcal{G}^{-}} .
$$

Since $P_{\mathcal{G}^{-}}=P_{\mathcal{G}^{n}}$ on $\mathcal{G}_{n}$ we have

$$
\begin{aligned}
E_{Q}\left\{h_{\mathcal{G}_{n}}\left(Q_{\mathcal{G}^{n}} \mid P_{\mathcal{G}^{n}}\right)\right\} & =E_{Q}\left\{h_{\mathcal{G}_{n}}\left(Q_{\mathcal{G}^{n}} \mid P_{\omega}^{n, k}\right)\right\}+E_{Q}\left\{\log z_{1 \wedge \tau_{k}}^{n}\right\} \geq E_{Q}\left\{\log z_{1 \wedge \tau_{k}}^{n}\right\} \\
& =\frac{1}{2} \sum_{i \in \Lambda_{n}} E_{Q} \int_{0}^{\tau_{k}} \beta^{2}\left(\theta_{i, s} \omega\right) d s
\end{aligned}
$$

and the conclusion follows by letting $k \rightarrow \infty$ and using the Monotone Convergence Theorem.

Proof of Theorem 2. Using (5.1), it is enough to show that $\beta(\omega)=\mathbf{b}(\omega) Q$-a.s.. Note that

$$
E_{Q}\left\{\int_{0}^{1} \mathbf{b}\left(\theta_{0, t} \omega\right) d \tilde{B}_{0}(t)-\frac{1}{2} \mathbf{b}^{2}(\omega)\right\}=E_{Q}\left\{\beta(\omega) \mathbf{b}(\omega)-\frac{1}{2} \mathbf{b}^{2}(\omega)\right\} .
$$

Thus, by (5.2)

$$
0=\mathcal{H}^{\mathbf{b}}(Q) \geq \frac{1}{2} E_{Q}\left\{(\beta(\omega)-\mathbf{b}(\omega))^{2}\right\}
$$

and the conclusion follows. 


\section{The case of Markovian gradient systems}

Some of the results in the previous sections can be strengthen in the case of gradient systems. We recall that a system on $\mathbb{R}^{\mathbb{Z}^{d}}$ is said to be gradient if $\mathbf{b}(\omega)=b(\omega(0))$, where

$$
b(x)=\frac{\partial}{\partial x_{0}} \sum_{j \in \mathbb{Z}^{d}} \psi\left(\theta_{j} x\right)
$$

for a local $\mathcal{C}^{2}$ function $\psi$. We also assume that the first and second derivatives of $\psi$ are bounded.

It is not restrictive to assume $\psi(x)=\psi\left(x_{\mathcal{L}}\right)$, where $\mathcal{L}$ is a symmetric finite subset of $\mathbb{Z}^{d}$ around the origin. Thus $b$ is $\Lambda_{0}$-local where we can choose $\Lambda_{0}=\left\{i \in \mathbb{Z}^{d}:(i+\mathcal{L}) \cap \mathcal{L} \neq \emptyset\right\}$, that is also symmetric around the origin. By symmetry, it follows that $\{0\}^{+}=\Lambda_{0}$.

Lemma 9. If the system is gradient then $\mathcal{H}(Q)<+\infty$ for every $Q \in \mathcal{G}_{S}\left(\Pi^{H}\right)$.

Proof. Consider the Hamiltonian

$$
H_{\{0\} \times] 0,2[}(\omega)=-\sum_{i \in \Lambda_{0}}\left[\int_{0}^{2} \mathbf{b}\left(\theta_{i, t} \omega\right) d \tilde{B}_{i}(t)-\frac{1}{2} \int_{0}^{2} \mathbf{b}^{2}\left(\theta_{i, t} \omega\right) d t\right] .
$$

Following a decoupling technique used in [6], for $i \in \Lambda_{0}$ we define

$$
\tilde{b}_{i}(x)=\frac{\partial}{\partial x_{i}} \sum_{j \in \mathbb{Z}^{d}} \psi\left(\theta_{j} x\right)
$$

We claim that, for all $i \in \Lambda_{0}$, the difference $\tilde{b}_{i}(x)-b\left(\theta_{i}(x)\right)$ is independent of $x_{0}$. To see this, noting that if $j \notin \mathcal{L}$ then $\psi\left(\theta_{j} x\right)$ does not depend on $x_{0}$, it is enough to show that

$$
\frac{\partial}{\partial x_{0}}\left(\frac{\partial}{\partial x_{i}} \sum_{j \in \mathbb{Z}^{d}} \psi\left(\theta_{j} x\right)-b\left(\theta_{i} x\right)\right)=0
$$

for every $i \in \Lambda_{0}$. Indeed

$$
\begin{aligned}
& \frac{\partial}{\partial x_{0}}\left(\frac{\partial}{\partial x_{i}} \sum_{j \in \mathbb{Z}^{d}} \psi\left(\theta_{j} x\right)-b\left(\theta_{i} x\right)\right) \\
& =\frac{\partial}{\partial x_{0}} \sum_{j \in \mathbb{Z}^{d}}\left(\frac{\partial \psi}{\partial x_{i-j}}\right)\left(\theta_{j} x\right)-\frac{\partial}{\partial x_{0}} \sum_{j \in \mathbb{Z}^{d}}\left(\frac{\partial \psi}{\partial x_{-j}}\right)\left(\theta_{i+j} x\right) \\
& =\sum_{j \in \mathbb{Z}^{d}}\left(\frac{\partial^{2} \psi}{\partial x_{-j} \partial x_{i-j}}\right)\left(\theta_{j} x\right)-\sum_{j \in \mathbb{Z}^{d}}\left(\frac{\partial^{2} \psi}{\partial x_{-i-j} \partial x_{-j}}\right)\left(\theta_{i+j} x\right) \equiv 0 .
\end{aligned}
$$


Having established (6.1), we have two equivalent forms for the local specification $\Pi_{\{0\} \times] 0,2[}^{H}$ :

$$
\begin{aligned}
& \Pi_{\{0\} \times] 0,2[}^{H}\left(\omega, d \omega^{\prime}\right)=\frac{1}{Z_{\{0\} \times] 0,2[}^{H}(\omega)} \exp \left[-H_{\{0\} \times] 0,2[}\left(\omega^{\prime}\right)\right] \Pi_{\{0\} \times] 0,2[}^{0}\left(\omega, d \omega^{\prime}\right) \\
& =\frac{1}{\tilde{Z}_{\{0\} \times] 0,2[}^{H}(\omega)} \exp \left\{\sum _ { i \in \Lambda _ { 0 } } \left[\int _ { 0 } ^ { 2 } \tilde { b } _ { i } ( \omega ^ { \prime } ( t ) ) \left(d \omega_{i}^{\prime}(t)\right.\right.\right. \\
& \left.\left.\left.\quad+\frac{1}{2} \varphi^{\prime}\left(\omega_{i}^{\prime}(t)\right) d t\right)-\frac{1}{2} \int_{0}^{2} b^{2}\left(\omega_{i}^{\prime}(t)\right) d t\right]\right\} \Pi_{\{0\} \times] 0,2[}^{0}\left(\omega, d \omega^{\prime}\right) .
\end{aligned}
$$

Letting $k(x)=\sum_{j \in \mathbb{Z}^{d}} \psi\left(\theta_{j} x\right)$, we note that $k(x)=k\left(x_{\Lambda_{0}}\right)$ and $\tilde{b}_{i}=\frac{\partial}{\partial x_{i}} k(x)$ for $i \in \Lambda_{0}$. Therefore, by Ito's rule,

$$
\begin{aligned}
\sum_{i \in \Lambda_{0}} \int_{0}^{2} \tilde{b}_{i}\left(\omega^{\prime}\right)\left(d \omega_{i}^{\prime}(t)+\frac{1}{2} \varphi^{\prime}\left(\omega_{i}^{\prime}(t)\right) d t\right)= & k\left(\omega^{\prime}(2)\right)-k\left(\omega^{\prime}(0)\right) \\
& -\frac{1}{2} \sum_{i \in \Lambda_{0}} \int_{0}^{2} \frac{\partial^{2}}{\partial x_{i}^{2}} k\left(\omega^{\prime}(t)\right) d t .
\end{aligned}
$$

Identities (6.2), (6.3) and the uniform boudedness of $\psi$ imply that

$$
\left|\log \frac{\Pi_{\{0\} \times] 0,2[}^{H}\left(\omega, d \omega^{\prime}\right)}{\Pi_{\{0\} \times] 0,2[}^{0}\left(\omega, d \omega^{\prime}\right)}\right| \leq C_{0}
$$

for some constant $C_{0}>0$ independent of both $\omega$ and $\omega^{\prime}$.

Consider now $Q \in \mathcal{G}_{S}\left(\Pi^{H}\right)$. Recalling that $\mathcal{B}^{-} \subset \hat{\mathcal{F}}_{\{0\} \times] 0,2[}$, we have

$$
\begin{aligned}
& \left.\left.\left|\log \frac{d Q_{\mathcal{B}^{-}}}{d W_{\omega_{0}(0)}}\right|_{\mathcal{B}_{1}}(\omega)|\leq| \log \frac{d Q_{\hat{\mathcal{F}}_{\{0\} \times] 0,2[}}}{d W_{0, \omega_{0}(0)}^{2, \omega_{0}(2)}}\right|_{\mathcal{B}_{1}}(\omega)|+| \log \frac{d W_{0, \omega_{0}(0)}^{2, \omega_{0}(2)}}{d W_{\omega_{0}(0)}}\right|_{\mathcal{B}_{1}}(\omega) \mid \\
& \leq\left|\log \frac{d \Pi_{\{0\} \times] 0,2[}^{H}}{d \Pi_{\{0\} \times] 0,2[}^{0}}(\omega)\right|+\left|\log \frac{d W_{0, \omega_{0}(0)}^{2, \omega_{0}(2)}}{d W_{\omega_{0}(0)}}\right|_{\mathcal{B}_{1}}(\omega) \mid .
\end{aligned}
$$

By (6.4), the first summand in (6.5) is uniformly bounded. Under Assumption A2, also the second summand in (6.5) is uniformly bounded, so that clearly

$$
\mathcal{H}(Q)=E_{Q}\left(h_{\mathcal{B}_{1}}\left(Q_{\mathcal{B}^{-}} \mid W_{\omega_{0}(0)}\right)\right)=E_{Q}\left(\left.\log \frac{d Q_{\mathcal{B}^{-}}}{d W_{\omega_{0}(0)}}\right|_{\mathcal{B}_{1}}\right)
$$

is finite.

Under Assumption A1, it is enough to show that

$$
E_{Q}\left(\left|\log \frac{d W_{0, \omega_{0}(0)}^{2, \omega_{0}(2)}}{d W_{\omega_{0}(0)}}\right|_{\mathcal{B}_{1}} \mid\right)<+\infty
$$


But, by the estimate (4.17), we have

$$
\left|\log \frac{d W_{0, \omega_{0}(0)}^{2, \omega_{0}(2)}}{d W_{\omega_{0}(0)}}\right|_{\mathcal{B}_{1}} \mid \leq A^{\prime}\left(\omega_{0}^{2}(0)+\omega_{0}^{2}(1)+\omega_{0}^{2}(2)+1\right) .
$$

The fact that the r.h.s. of (6.6) is in $L^{1}(Q)$ is shown in (4.18).

With the given potential $\psi$ we can define, for $\Lambda \subset \mathbb{Z}^{d}$ finite, the Hamiltonian

$$
h_{\Lambda}(x)=-2 \sum_{i:\left(i+\Lambda_{0}\right) \cap \Lambda \neq \emptyset} \psi\left(\theta_{i} x\right) \quad\left(h_{\{0\}}(x)=-2 k(x)\right)
$$

and the local specifications on $\mathbb{R}^{\mathbb{Z}^{d}}$

$$
\Pi_{\Lambda}^{h}\left(x, d x^{\prime}\right)=\frac{1}{Z_{\Lambda}^{h}(x)} \exp \left[-h_{\Lambda}\left(x^{\prime}\right)\right] \otimes_{i \in \Lambda} \lambda\left(d x_{i}\right) \otimes \otimes_{i \notin \Lambda} \delta_{x_{i}}\left(d x_{i}^{\prime}\right)
$$

where $\lambda(d x)=e^{-\varphi(x)} d x$. We let $\mathcal{G}\left(\Pi^{h}\right)$ denote the set of Gibbs measures for the specifications in (6.7), and $\mathcal{G}_{S}\left(\Pi^{h}\right)$ the subset of those that are invariant for the space-shifts $\left(\theta_{i}\right)_{i \in \mathbb{Z}^{d}}$.

Proposition 4. For $Q \in \mathcal{P}_{S}(\Omega)$, we let $Q_{0}$ denote its one-time marginal, i.e.

$$
Q_{0}(E)=Q(\{\omega: \omega(0) \in E\}),
$$

where $E$ is a Borel subset of $\mathbb{R}^{\mathbb{Z}^{d}}$.

Then the map $Q \rightarrow Q_{0}$ is a bijection between $\mathcal{G}_{S}\left(\Pi^{H}\right)$ and $\mathcal{G}_{S}\left(\Pi^{h}\right)$.

Proof. Let $\mathcal{S}$ be the set of stationary weak solutions of equation (2.4). It is shown in [9], Theorem 3.10, that the stationary measures for (2.4) that are also invariant for the space shifts $\left(\theta_{i}\right)_{i \in \mathbb{Z}^{d}}$ are exactly the elements of $\mathcal{G}_{S}\left(\Pi^{h}\right)$. This can be rephrased by saying that the map $Q \rightarrow Q_{0}$ is a bijection between $\mathcal{S} \cap \mathcal{P}_{S}(\Omega)$ and $\mathcal{G}_{S}\left(\Pi^{h}\right)$. On the other hand, by Proposition 1, Theorems 1, 2 and Lemma 9 we have that $\mathcal{S} \cap \mathcal{P}_{S}(\Omega)=\mathcal{G}_{S}\left(\Pi^{H}\right)$.

In particular, if the potential $\psi$ is bounded with an $L^{\infty}$-norm small enough, together with the norm of its first and second derivatives, then the Gibbs measures are small perturbations of a free field, and by [18] Theorem 1 page $105, \mathcal{G}_{s}\left(\Pi^{h}\right)$ contains exactly one element (which furthermore admits a cluster expansion). So $\mathcal{G}_{s}\left(\Pi^{H}\right)$ is also reduced to a unique space-time Gibbs measure.

Acknowledgements. S.R. thanks the Dipartimento di Matematica, Politecnico di Milano, for the very kind hospitality. 


\section{Appendix. Proof of Lemma 3}

We begin by proving part a. of Lemma 3. We first associate to the potential $\varphi(x)$ the function

$$
\tilde{\varphi}(x)=\varphi^{\prime \prime}(x)-\frac{1}{2} \varphi^{\prime}(x)^{2} .
$$

An elementary integration argument shows that, under Assumption A1 there are constants $A_{1}, A_{2}>0$ and $B_{1}, B_{2} \in \mathbb{R}$ such that, for all $x \in \mathbb{R}$,

$$
-A_{1} x^{2}+B_{1} \leq \tilde{\varphi}(x) \leq-A_{2} x^{2}+B_{2} .
$$

Note that, if we consider the quadratic potential $\varphi^{*}(x)=\frac{1}{2} a x^{2}, a>0$, we obtain

$$
\tilde{\varphi^{*}}(x)=a-\frac{1}{2} a^{2} x^{2}
$$

Thus, by (7.1) and (7.2), we get that there are quadratic potentials $\varphi^{-}(x)=\frac{1}{2} a^{-} x^{2}$, $\varphi^{+}(x)=\frac{1}{2} a^{+} x^{2}$ and a constant $C>0$ such that

$$
\forall x \in \mathbb{R}, \quad \tilde{\varphi^{-}}(x)-C \leq \tilde{\varphi}(x) \leq \tilde{\varphi^{+}}(x)+C .
$$

Upper bounds for $q_{t}(x, y)$. The key idea for obtaining the desired bounds on $q_{t}(x, y)$ is to use the following representation (see [28], Theorem 7.5.13):

$$
q_{t}(x, y)=e^{\frac{1}{2} \varphi(x)+\frac{1}{2} \varphi(y)} \gamma_{t}(x-y) E_{0, y}^{t, x}\left[\exp \left(\int_{0}^{t} \frac{1}{4} \tilde{\varphi}\left(\omega_{s}\right) d s\right)\right] \int e^{-\varphi(z)} d z,
$$

where

$$
\gamma_{t}(\xi)=\frac{1}{\sqrt{2 \pi t}} e^{-\frac{\xi^{2}}{2 t}}
$$

and $E_{0, y}^{t, x}$ is the expectation with respect to the Brownian Bridge with endpoints $(0, y)$ and $(t, x)$. By the upper bound in (7.3) and (7.4) we obtain

$$
q_{t}(x, y) \leq K e^{\frac{1}{2} \varphi(x)-\frac{1}{2} \varphi^{+}(x)} e^{\frac{1}{2} \varphi(y)-\frac{1}{2} \varphi^{+}(y)} e^{C t} q_{t}^{+}(x, y),
$$

where $K$ is a positive constant and

$$
q_{t}^{+}(x, y)=\frac{1}{\sqrt{1-e^{-a^{+} t}}} \exp \left[-\frac{1}{2 \sigma_{t}^{2}}\left[m_{t}^{2} x^{2}+m_{t}^{2} y^{2}-2 m_{t} x y\right]\right],
$$

with

$$
m_{t}=e^{-\frac{a^{+}}{2} t}, \quad \sigma_{t}^{2}=\frac{1}{a^{+}}\left(1-e^{-a^{+} t}\right)
$$


is the transition density associated with the quadratic potential $\varphi^{+}$. It is easily seen that

$$
q_{t}^{+}(x, y) \leq \frac{1}{\sqrt{1-e^{-a^{+} t}}} \exp \left[\frac{m_{t}}{\sigma_{t}^{2}} x y\right] \leq \frac{1}{\sqrt{1-e^{-a^{+} t}}} \exp \left[\frac{m_{t}}{2 \sigma_{t}^{2}}\left(x^{2}+y^{2}\right)\right] .
$$

Since $m_{t} \rightarrow 0$ as $t \rightarrow+\infty$, there is a $T>0$ such that

$$
e^{-\frac{1}{2} \varphi^{+}(x)} \exp \left[\frac{m_{T}}{2 \sigma_{T}^{2}} x^{2}\right] \leq e^{-\frac{1}{4} \varphi^{+}(x)} .
$$

Plugging this in (7.5), we obtain

$$
q_{T}(x, y) \leq M e^{g(x)} e^{g(y)}
$$

for a suitable $M>0$ and

$$
g(x)=\frac{1}{2} \varphi(x)-\frac{1}{4} \varphi^{+}(x)
$$

In what follows we denote by $\mu$ the probability measure $\frac{e^{-\varphi(x)}}{\int e^{-\varphi(y)} d y} d x$. By abuse of notation, we let

$$
\mu(x)=\frac{e^{-\varphi(x)}}{\int e^{-\varphi(y)} d y} .
$$

By Assumption A1, $\mu$ has Gaussian tails. It follows ([28], Corollary 7.5.38) that the semigroup

$$
T_{t} f(x)=\int f(\xi) p_{t}(\xi, x) d \xi
$$

is contractive in $L^{2}(\mu)$, i.e. there is $\alpha>0$ (the spectral Gap) such that for all $f \in L^{2}(\mu)$

$$
\left\|T_{t} f-\int f d \mu\right\|_{L^{2}(\mu)} \leq e^{-\alpha t}\left\|f-\int f d \mu\right\|_{L^{2}(\mu)} .
$$

As a consequence, for $f \in L^{2}(\mu)$

$$
\left|E_{W}\left(f\left(x_{t}\right) f\left(x_{0}\right)\right)-\left(\int f d \mu\right)^{2}\right| \leq e^{-2 \alpha t}\|f\|_{L^{2}(\mu)}^{2} .
$$

Now, using the fact that, for $g$ defined in (7.7), $e^{g} \in L^{2}(\mu)$, for $t>3 T$ we have

$$
\begin{aligned}
q_{t}(x, y) & =\int q_{T}(x, z) p_{t-2 T}(z, w) q_{T}(w, y) \mu(w) d z d w \\
& \leq M^{2} e^{g(x)+g(y)} \int e^{g(z)} e^{g(w)} p_{t-2 T}(z, w) d z d w \\
& \leq M^{2} e^{g(x)+g(y)}\left\|e^{g}\right\|_{L^{2}(\mu)}^{2}\left(1+e^{-2 \alpha T}\right) .
\end{aligned}
$$


Since the last bound is uniform for $t>2 T$ and $g(x)$ has at most quadratic growth, the upper bound in (4.3) follows easily.

Lower bounds for $q_{t}(x, y)$. We proceed as in the upper bound. By (7.5) it is easily seen that for a given $T>0$ there exist $M, a>0$ such that

$$
q_{T}(x, y) \geq M e^{-a\left(x^{2}+y^{2}\right)} .
$$

We now proceed as in the upper bound, with $g(x)=-a x^{2}$.

We now prove part b. of Lemma 3 and work under Assumption A2. Define

$$
q_{t} * q_{s}(w, z)=\int q_{t}(w, y) q_{s}(y, z) \mu(y) d y .
$$

Then, for any $a \in\left[0, \frac{t}{2}\right]$

$$
\begin{gathered}
\sup _{w, z \in \mathbb{R}}\left|q_{t}(w, z)-1\right|=\sup _{w, z \in \mathbb{R}}\left|q_{a} *\left(q_{t-2 a}-1\right) * q_{a}(w, z)\right| \\
\quad \leq \sup _{z \in \mathbb{R}} \int \sup _{w \in \mathbb{R}} q_{a} *\left|q_{t-2 a}-1\right|(w, y) q_{a}(y, z) \mu(y) d y .
\end{gathered}
$$

By Theorem 1.4 in [14] the semigroup $T_{t}$, under Assumption A2, is ultracontractive, i.e. it maps $L^{2}(\mu)$ into $L^{\infty}(\mu)$; so there exists $C_{1}(a)>0$ such that

$$
\sup _{w \in \mathbb{R}} q_{a} *\left|q_{t-2 a}-1\right|(w, y) \leq C_{1}(a)\left\|\left(q_{t-2 a}-1\right)(\cdot, y)\right\|_{L^{2}(\mu)} .
$$

So

$$
\begin{aligned}
\sup _{w, z \in \mathbb{R}}\left|q_{t}(w, z)-1\right| & \leq C_{1}(a) \sup _{z \in \mathbb{R}} \int q_{a}(y, z)\left\|\left(q_{t-2 a}-1\right)(\cdot, y)\right\|_{L^{2}(\mu)} \mu(d y) \\
& \leq C_{1}^{2}(a)\|y \rightarrow\|\left(q_{t-2 a}-1\right)(\cdot, y)\left\|_{L^{2}(\mu)}\right\|_{L^{2}(\mu)} .
\end{aligned}
$$

Now, is it known that ultracontractivity implies $L^{2}$-contractivity. Thus, denoting again by $\alpha$ the spectral gap,

$$
\begin{aligned}
\left\|\left(q_{t-2 a}-1\right)(\cdot, y)\right\|_{L^{2}(\mu)} & =\left\|q_{t-2 a}(\cdot, y)-\int q_{t-2 a}(x, y) \mu(d x)\right\|_{L^{2}(\mu)} \\
& \leq e^{-(t-3 a) \alpha}\left\|q_{a}(\cdot, y)-1\right\|_{L^{2}(\mu)}
\end{aligned}
$$

which implies

$$
\sup _{w, z \in \mathbb{R}}\left|q_{t}(w, z)-1\right| \leq C_{1}^{2}(a) e^{-(t-3 a) \alpha} \sqrt{\iint\left(q_{a}(x, y)-1\right)^{2} \mu(d x) \mu(d y)}
$$

which converges exponentially to zero as $t$ tends to infinity. It allows to conclude that

$$
\lim _{t \rightarrow+\infty} \sup _{w, z \in \mathbb{R}}\left|q_{t}(w, z)-1\right|=0
$$




\section{References}

1. Courrège, Ph., Renouard, P.: Oscillateurs anharmoniques, mesures quasi-invariantes sur $C(R, R)$ et théorie quantique des champs en dimension 1, Astérisque 22-23 Soc. Math. France., Paris (1975)

2. Cattiaux, P., Roelly, S., Zessin, H.: Une approche Gibbsienne des diffusions Browniennes infini-dimensionnelles, Probab. Th. Rel. Fields., 104, 147-179 (1996)

3. Dai Pra, P.: Space-time large deviations for interacting particle systems, Comm. Pure and Appl. Math., Vol. XLVI 387-422 (1993)

4. Dai Pra, P.: Large deviations and stationary measures for interacting particle systems, Stoch. Proc. Appl., 48, 9-30 (1993)

5. Dai Pra, P., Roelly, S.: Cluster expansion for infinite-dimensional Brownian diffusions with non-regular drift, in preparation

6. Deuschel, J.D.: Non-linear smoothing of infinite-dimensional diffusion processes, Stochastics., Vol. 19 237-261 (1986)

7. Deuschel, J.D.: Infinite-dimensional diffusion processes as Gibbs measures on $C[0,1]^{\mathbb{Z}^{d}}$, Probab. Th. Rel. Fields., 76, 325-340 (1987)

8. Föllmer, H.: On entropy and information gain in random fields, Z. Wahrsch. Verw. Geb., 26, 207-217 (1973)

9. Fritz, J.: Stationary measures of stochastic gradient systems, infinite lattice models, Z. Warsch. Verw. Geb., 59, 479-490 (1982)

10. Föllmer, H., Wakolbinger, A.: Time reversal of infinite-dimensional diffusions, Stoch. Proc. Appl., 22, 59-77 (1986)

11. Ignatyuk, I.A., Malyshev, V.A., Sidoravicius, V.: Convergence of the stochastic quantization method I*, Theory Prob. Appl., 37-2 (1990)

12. Jamison, B.: Reciprocal processes, Z. Warsch. Verw. Geb., 30, 65-86 (1974)

13. Jona-Lasinio, G., Sénéor, R.: Study of Stochastic Differential Equations by Constructive Methods I*, J. Stat. Phys., 83, 5-6 1109-1148 (1996)

14. Kavian, O., Kerkyacharian, G., Roynette, B.: Quelques remarques sur l'ultracontractivité, J. Func. Anal., 111, 155-196 (1993)

15. Künsch, H.R.: Almost sure entropy and the variational principle for random fields with unbounded state space, Z. Wahrsch. Verw. Geb., 58, 69-85 (1981)

16. Lebowitz, J.L., Presutti, E.: Statistical Mechanics of systems of unbounded spins, Comm. Math. Phys., 50, 195-218 (1976)

17. Macris, N., Martin, Ph.A., Pulé, J.V.: Diamagnetic currents, Comm. Math. Phys., 117, 215-241 (1988)

18. Malyshev, V.A., Minlos, R.A.: Gibbs Random Fields, Cluster expansions, Mathematics and Its Applications., Vol. 44, Kluwer Ac. Pub. (1991)

19. Minlos, R.A., Roelly, S., Zessin, H.: Gibbs states on space-time, Potential Analysis, 13, 367-408 (2000)

20. Minlos, R.A., Verbeure, A., Zagrebnov, V.: A quantum cristal model in the light mass limit: Gibbs states, Rev. Math. Phys. Vol. 12(7), 981-1032 (2000)

21. Osada, H., Spohn, H.: Gibbs measures relative to Brownian motion, Ann. Prob., 27(3), 1183-1207 (1999)

22. Preston, C.: Random fields, L.N. in Math., 534, Springer (1976)

23. Robinson, D.W., Ruelle, D.: Mean entropy of states in classical statistical mechanics, Comm. Math. Phys., 5, 288-300 (1967)

24. Roelly, S., Zessin, H.: Sur la mécanique statistique d'une particule brownienne sur le tore, Séminaire de Probabilités XXV, L.N. in Math., 1485, Springer 291-310 (1991)

25. Royer, G.: Une initiation aux inegalités de Sobolev logarithmiques, Cours Spécialisés, Soc. Math. France., Paris (1999) 
26. Royer, G., Yor, M.: Représentation intégrale de certaines mesures quasi-invariantes sur $C(R)$; mesures extrémales et propriété de Markov, Ann. Inst., Fourier (Grenoble) 26-2 7-24 (1976)

27. Shiga, T., Shimizu, A.: Infinite dimensional stochastic differential equations and their applications, J. Math. Kyoto Univ., 20(3), 395-416 (1980)

28. Stroock, D.W.: Probability Theory, An analytic view, Cambridge University Press (1993) 